\title{
Alpha-D-Galactosidase does not Interfere with Trimebutine Oral Pharmacokinetics in Mexican Healthy Volunteers
}

\author{
Peñaloza-Becerra CA ${ }^{1}$, Ortega-Escamilla E¹, Vasquez-Vasquez JE¹, Marcelín-Jiménez G², Ángeles AP², García-González A², Laguna-Leyte JS ${ }^{3}$, \\ Koretzky SG${ }^{3}$, Batista-Dieguéz $\mathrm{D}^{3}$ and López-Sánchez $\mathbf{P}^{4^{*}}$
}

${ }^{1}$ Centro A.F. de Estudios Tecnológicos, S.A. de C.V., Ciudad de México, México

${ }^{2}$ Global Bioanalytical Consulting, S.C., Ciudad de México, México

${ }^{3}$ Pharmometrica (Pharmet, S.A. de C.V.), Ciudad de México, México

${ }^{4}$ Escuela Superior de Medicina, Instituto Politécnico Nacional, Ciudad de México, México

\begin{abstract}
Gas production is a common symptom in bowel affections. There are different formulations to improve general symptoms, including motility regulators, such as trimebutine, and surfactants, such as simethicone, or both. These approaches, however, do not affect gas production. Methane, hydrogen, carbon dioxide, and water are generated in the intestines due to action of bacterial flora on non-digestible carbohydrates from the diet. The unfolding of these carbohydrates by specific enzymes promises greater improvement of symptomatology. Alpha-D-Galactosidase degrades these carbohydrates from diet. It is not known whether the addition of this enzyme modifies trimebutine pharmacokinetics. Thus, our aim was to assess whether the addition of Alpha-D-Galactosidase to a commercial formulation alters trimebutine oral pharmacokinetics. We conducted a controlled, cross-over, randomized, simpleblind, two-period, two-treatment, and two-sequence clinical trial on 30 healthy Mexican volunteers, receiving a single dose of reference product and test product. Pharmacokinetics and safety of usage were obtained. We measured $\mathrm{N}$-desmethyl-trimebutine, the major metabolite of trimebutine. We showed that addition of galactosidase does not modify any pharmacokinetic parameter significantly. Safety of the subjects was not affected. We conclude that alpha-D-Galactosidase does not modify oral pharmacokinetics of trimebutine, rendering this approach suitable for commercial use in indicated bowel affections.
\end{abstract}

Keywords: Alpha-D-galactosidase; Trimebutine; Simethicone; Irritable bowel syndrome; Gas production; Mexican population; Healthy volunteers

\section{Introduction}

Gas production in the intestine of humans is related with many different processes, including diet, intestinal flora, and pathologic stages. Additionally, the social implications of gas production may be embarrassing due to flatulence and odour problems [1]. Because of these interactions, a definite treatment is difficult to establish, given that gas-generating processes are different in every patient. In Mexico, as in the majority of Western countries, there are different pathologies associated with intestinal gas production. The estimated prevalence of this symptom is between 10 and $20 \%$ in general population [2]. Main causes include Irritable Bowel Syndrome (IBS), lactose intolerance or to other carbohydrates, acute and chronic non-infectious colitis, and other non-specific pathologies without evidence of neoplastic, inflammatory, metabolic, or anatomic affections [3]. In addition to gas production, in the majority of these diseases, other symptoms require being taken into account in order to improve patient conditions. The majority of times, aside from a disease-specific base treatment, an antispasmodic agent is required to alleviate associated pain, while at other times, a prokinetic agent to relieve motility disorders is indicated or, simply, a surfactant to facilitate gas elimination from the bowels. Moreover, in many cases, a combined treatment is needed to achieve the therapeutic goals. In this regard, several combinations have been developed and are available worldwide. One of these combinations is the association of Trimebutine, a parasympatholytic drug directed toward improving gastrointestinal motility, which is widely absorbed in small bowel and suffers an extensive first-pass metabolism [4], and Simethicone, an inert, non-absorbable silicone dioxide polymer with tensoactive and foam-dissolving properties. Although clinical improvement has been reported in many cases using this combination, flatulence continues to remain a complaint from patients [5]. Given the Latin-American diet, which is rich in Non-Digestible Carbohydrates (NDC) such as raffinose, stachiose, and verbascose (carbohydrates from leguminosae such as beans, peas, soy, lentils, and cereals such as rice, oat, corn, or wheat), gas generation due to the action of microorganisms upon these is frequent. Therefore, it is highly suitable to generate new formulations that aid in processing these NDC. Alpha-D-Galactosidase (AG) is an enzyme produced by different molds and bacteria and that breaks down different non-absorbable oligosaccharides. This property renders it eligible to be administered associated to other drugs and improve the symptoms of gas production [6]. Oral administration of AG does not have systemic absorption; it remains in the large intestine and the first portion of duodenum, where it exerts its effect. It is necessary, however, to first describe whether the addition of AG does not alter the pharmacokinetics of known compounds in combinations widely used in the previously mentioned diseases [4,7]. Therefore, the aim of this work was to evaluate whether or not the oral pharmacokinetics of Trimebutine, as utilized commercially in combination with Simethicone, was not modified by the co-formulation and administration of AG in a group of healthy Mexicans.

*Corresponding author: López-Sánchez $\mathrm{P}, \mathrm{MD}, \mathrm{PhD}$, Sección de Estudios de Posgrado e Investigación, Escuela Superior de Medicina del Instituto Politécnico Nacional, Plan de San Luis y Díaz Mirón s/n, Col. Casco de Sto. Tomás, C.P. 11340, Ciudad de México, México, Tel: +52-55-57296300; E-mail: pelosa651018@yahoo.com

Received May 17, 2017; Accepted June 09, 2017; Published June 16, 2017

Citation: Peñaloza-Becerra CA, Ortega-Escamilla E, Vasquez-Vasquez JE, Marcelín-Jiménez G, P.Angeles-Moreno AC, et al. (2017) Alpha-D-Galactosidase does not Interfere with Trimebutine Oral Pharmacokinetics in Mexican Healthy Volunteers. J Bioequiv Availab 9: 447-451. doi: 10.4172/jbb.1000342

Copyright: () 2017 Peñaloza-Becerra CA, et al. This is an open-access article distributed under the terms of the Creative Commons Attribution License, which permits unrestricted use, distribution, and reproduction in any medium, provided the original author and source are credited. 
Citation: Peñaloza-Becerra CA, Ortega-Escamilla E, Vasquez-Vasquez JE, Marcelín-Jiménez G, P.Angeles-Moreno AC, et al. (2017) Alpha-DGalactosidase does not Interfere with Trimebutine Oral Pharmacokinetics in Mexican Healthy Volunteers. J Bioequiv Availab 9: 447-451. doi: $10.4172 / j b b .1000342$

\section{Materials and Methods}

\section{Formulations}

The reference formulation was Libertrim SII tablets, containing 200 mg of Trimebutine maleate and $75 \mathrm{mg}$ of Simethicone (batch \#S1507506; expiration date, June 2017). The test product was Libertrim Alfa containing Trimebutine maleate $200 \mathrm{mg}$, Simethicone $75 \mathrm{mg}$, and 450 IU of AG (batch \#C1510783; expiration date, Dec. 2017). Both products were manufactured by Productos Científicos, S.A. de C.V. (Mexico City, Mexico). This work was carried out to obtain marketing authorization by the Mexican Health Ministry for the test product.

\section{Clinical design}

Volunteers: Thirty healthy Mexican volunteers participated in this study. Seventeen were females and 13 were males. Inclusion criteria were age between 18 and 55 years, Body Mass Index (BMI) between 18 and $27 \mathrm{~kg} / \mathrm{m}^{2}$, non-smokers or not having smoked at least $72 \mathrm{~h}$ prior to the study, normal clinical history and Electrocardiogram (EKG) study, laboratory values within $\pm 10 \%$ of normal mean values (blood biochemistry, hematology, urine analysis, and liver function), and negative for AIDS and hepatitis B and C and, in females, pregnancy tests. Exclusion criteria included allergic history to any component of formulation used, pregnancy, alcohol addiction history or consumption $24 \mathrm{~h}$ prior to the study, the use of any drug 2 weeks before the study (positive for the Rapid Drug Abuse Assay), and any serious health condition that would affect the performance of the study. Withdrawal criteria throughout the study comprised hypersensitivity reactions to any component of the formulations used, loss of two or more samples regarding $\mathrm{C}_{\max }$, diet transgression, or vomiting between administration times and 2 -fold $\mathrm{T}_{\max }$. Signed informed consent forms were obtained from each volunteer. All volunteers were medically monitored closely throughout the whole study. The protocol was revised and approved by the Global Bioanalytical Consulting Ethics Committee, and also was conducted in accordance with the principles stated in the Declaration of Helsinki and consistent with Good Clinical Practice (GCP), as defined by the International Conference on Harmonization (ICH) and in accordance with the ethical principles underlying European Union Directive 2001/20/EC and the United States Code of Federal Regulations, Title 21, Part 50 (21CFR50) [8].

Drug administration and sample collection: The trial design was controlled, cross-over, randomized, simple-blind (each product was blinded to the analytical investigator), two periods (length, $38 \mathrm{~h}$ per period, with 6 days of washout period in-between), two treatments (200 $\mathrm{mg}$ Trimebutine maleate/75 mg of Simethicone/450 IU of AG, or $200 \mathrm{mg}$ Trimebutine maleate/75 mg of Simethicone), with two administration sequences. We employed a single-dose design protocol with an entire length of 9 days. Treatment groups were balanced, that is, had the same number of volunteers randomly assigned to each administration sequence [9].

Volunteers presented the day prior to drug administration. They received dinner at 8:00 pm and fasted overnight (12 h). An indwelling cannula was set in place the following morning and a single dose of either tablet (200 mg Trimebutine maleate/75 mg of Simethicone/450 IU of AG, or $200 \mathrm{mg}$ Trimebutine maleat/75 mg of Simethicone) was taken with $250 \mathrm{~mL}$ of water at 8:00 am. Breakfast, lunch, and dinner were served $2.5,6.5$, and $12 \mathrm{~h}$ after dose administration, respectively. After having collected the last sample at 8:00 am on day 3, the volunteers left the research center and 6 days later ( 1 day prior to the second administration), volunteers re-entered in order to initiate period 2, according to the same schedule.

Approximately $6 \mathrm{~mL}$ was drawn for each blood sample through the cannula at $0 \mathrm{~h}$ (prior to administration) and $0.33,0.66,1.00,1.25$, $1.50,1.75,2.00,2.50,3.00,4.00,5.00,7.00,9.00,12.00$, and $24.00 \mathrm{~h}$ after dosing. Samples were collected in heparinized tubes and centrifuged at $4,000 \mathrm{rpm}$ for $5 \mathrm{~min}$ at room temperature. Plasma was collected in labeled cryovials and maintained frozen at $-70^{\circ} \mathrm{C}$ until chromatographic analysis.

\section{Safety assessment}

Safety assessments were performed through monitoring of the incidence and severity of Adverse Events (AE), physical examination findings, vital signs, and close observations of volunteers. Any complaint referred by subjects was registered for analysis.

\section{Sample preparation and Ultra Performance Liquid Chromatography-Tandem Mass Spectrometry (UPLC-MS/ MS) analysis}

$\mathrm{N}$-desmethyl-Trimebutine (NDT) is the main active metabolite of Trimebutine. Sample purification and chromatographic and mass spectrometric conditions were based on previously reported techniques [10]. Briefly, $200 \mu \mathrm{L}$ of plasma was added to Verapamil (Internal Standard [IS]), mixed with $600 \mu \mathrm{L}$ of cold acetonitrile, and centrifuged; supernatant was diluted 1:1 with mobile phase and $3 \mu \mathrm{L}$ was injected into the UPLC. Chromatographic system consisted of an Acquity equipment (Waters, Inc., Milford, MA, USA) coupled with a Xevo ${ }^{\text {sn }}$ TQMS tandem mass spectrometer (Micromass, Manchester, UK). Mobile phase was a mixture of aqueous formic acid (0.1\%) and acetonitrile $(70: 30 \mathrm{v} / \mathrm{v})$. Column was an Acquity UPLC $\mathrm{BEH}^{\mathrm{rw}} \mathrm{C} 18$ (Waters, Inc.) of $2.1 \times 50 \mathrm{~mm}$ and a particle size of $1.7 \mu \mathrm{m}$. Detection was through positive electrospray, employing the ionic transitions of $374.27>343.16$, and $455.41>165.07$ for NDT and IS, respectively.

Method was fully validated according U.S. Federal Drug Administration (FDA) guidelines and Mexican normativity, and probed to be linear within the range of $40-4,000 \mathrm{ng} / \mathrm{mL}$ of NDT, with precision and accuracy $[11,12]$.

\section{Pharmacokinetic analysis}

Pharmacokinetic parameters were calculated from analyzed plasma obtained from single dose-receiving volunteers, utilizing Phoenix WinNonlin ver. 6.4 software (Certara L.P., NJ, USA), considering a noncompartmental model.

Plasma elimination half-life $\left(t_{1 / 2}\right)$, Area Under the Curve (AUC) until the last measurable concentration $\left(\mathrm{AUC}_{0-\mathrm{t}}\right)$, area under curve was extrapolated to infinity $\left(\mathrm{AUC}_{0-\infty}\right)$, Mean Residence Time (MRT), and elimination constant $\left(\mathrm{k}_{\mathrm{e}}\right)$ were software outputs. Maximal observed concentration $\left(\mathrm{C}_{\max }\right)$ and time of $\mathrm{C}_{\max }\left(\mathrm{T}_{\max }\right)$ were obtained experimentally.

\section{Statistical analyses}

To evaluate fixed effects such as period, sequence, and formulation, we utilized Analysis of Variance (ANOVA) for a standard $2 \times 2$ crossover design. Possible pharmacokinetic modifications were evaluated using the Schuirmann two one-sided test to determine differences between $\mathrm{C}_{\max }, \mathrm{AUC}_{0-\mathrm{t}}$ and $\mathrm{AUC}_{0-\infty} . \mathrm{T}_{\max }, \mathrm{t}_{1 / 2}$, and MRT were compared through the Wilcoxon test. Statistical analyses were carried out using Phoenix 
Citation: Peñaloza-Becerra CA, Ortega-Escamilla E, Vasquez-Vasquez JE, Marcelín-Jiménez G, P.Angeles-Moreno AC, et al. (2017) Alpha-DGalactosidase does not Interfere with Trimebutine Oral Pharmacokinetics in Mexican Healthy Volunteers. J Bioequiv Availab 9: 447-451. doi: $10.4172 / j b b .1000342$

WinNonlin ver. 6.4 software (Certara L.P.) and MinitabTM ver. 16 software (Minitab, State College, PA, USA).

\section{Results}

\section{Subjects}

No study dropouts or withdrawals took place among the participants along the time that the protocol lasted. As shown in Table 1, volunteers comprised a homogeneous group, in which the average age was $28.93 \pm$ 8.45 years and mean BMI was $23.27 \pm 2.36$.

\section{Safety}

Regarding safety, both formulations were well tolerated by volunteers. No major AE were reported. In period 1, only one female subject reported rhinorrhea for a short period of time, with no treatment needed, the condition disappearing spontaneously. In period 2, a female volunteer reported slight dizziness, requiring only rest and medical observation.

\section{Pharmacokinetics}

Concentration-time value profiles for the 30 subjects who took both formulations are depicted in Figure 1. As can be clearly observed, determination of the Trimebutine major metabolite, N-desmethyl-

\begin{tabular}{|c|c|c|c|c|c|c|}
\hline Variable & Mean & SD & \%CV & Min value & Max value & Median \\
\hline Age $($ years $)$ & 28.93 & 8.45 & 29.20 & 18.00 & 48.00 & 27.00 \\
\hline Height $(\mathrm{m})$ & 1.63 & 0.10 & 6.22 & 1.46 & 1.82 & 1.63 \\
\hline Weight $(\mathrm{kg})$ & 62.05 & 9.42 & 15.18 & 43.40 & 82.90 & 62.60 \\
\hline BMI $\left(\mathrm{kg} / \mathrm{m}^{2}\right)$ & 23.27 & 2.36 & 10.16 & 18.30 & 26.90 & 23.20 \\
\hline
\end{tabular}

Subjects included in the study were 17 females (56.67\%) and 13 males $(43.33 \%)$ SD: Standard Deviation; BMI: Body Mass Index; CV: Coefficient of Variation Table 1: Anthropometric description of studied population.
Trimebutine, that is responsible for part of the pharmacological action in the studied formulations, is practically identical in both preparations. Co-formulation with AG appears not to modify or alter the pharmacokinetic parameters in the test product compared with the reference product. In this regard, $C_{\text {max }}$ were $1,882.079 \pm 548.692 \mathrm{ng} /$ $\mathrm{mL}$ and $1,926.538 \pm 620.355 \mathrm{ng} / \mathrm{mL}$ for reference and test products, respectively. In the same manner, $\mathrm{k}_{\mathrm{e}}\left(0.311 \pm 0.097 \mathrm{~h}^{-1}\right.$, reference, vs. $0.298 \pm 0.096 \mathrm{~h}^{-1}$, test $)$ and $\mathrm{T}_{\max }(1.283 \pm 0.327 \mathrm{~h}$, reference vs. $1.320 \pm$ $0.538 \mathrm{~h}$, test) were not different between themselves. This suggests that AG does not interfere either with the absorption or with the elimination processes of Trimebutine (Table 2).

\section{Discussion and Conclusion}

In this work, we evaluated the effect of AG upon Trimebutine pharmacokinetic parameters to assess probable pharmacokinetic and/ or formulation interactions as a previous step for the clinical assay of this new formulation. To our knowledge, this is the first report of AG combined with Trimebutine and Simethicone in a formulation designated to relieve symptoms related with different bowel diseases, in which common data comprise are gas production and bloating. There are, indeed, several formulations available for the treatment of pathologies such as IBS, lactose intolerance, or the intolerance of other carbohydrates, acute and chronic non-infectious colitis, Fabry's disease, and other bowel diseases [13]. Common therapeutic approaches often include avoiding the consumption of gas-associated food, such as dairy, cereals, legumes, fruits, and vegetables, along with prokinetic or antispasmodic drugs, with the idea of reducing pain and regulating intestinal transit, thus accelerating the elimination of gas-producing elements. Simethicone, an inert surfactant, is often associated in order to break up gas bubbles in order to facilitate gas elimination, therefore diminish symptom severity [5]. However, gas production is not affected by these formulations. In this regard, this novel formulation that includes AG could improve this point in the general stage (Table 3 ).

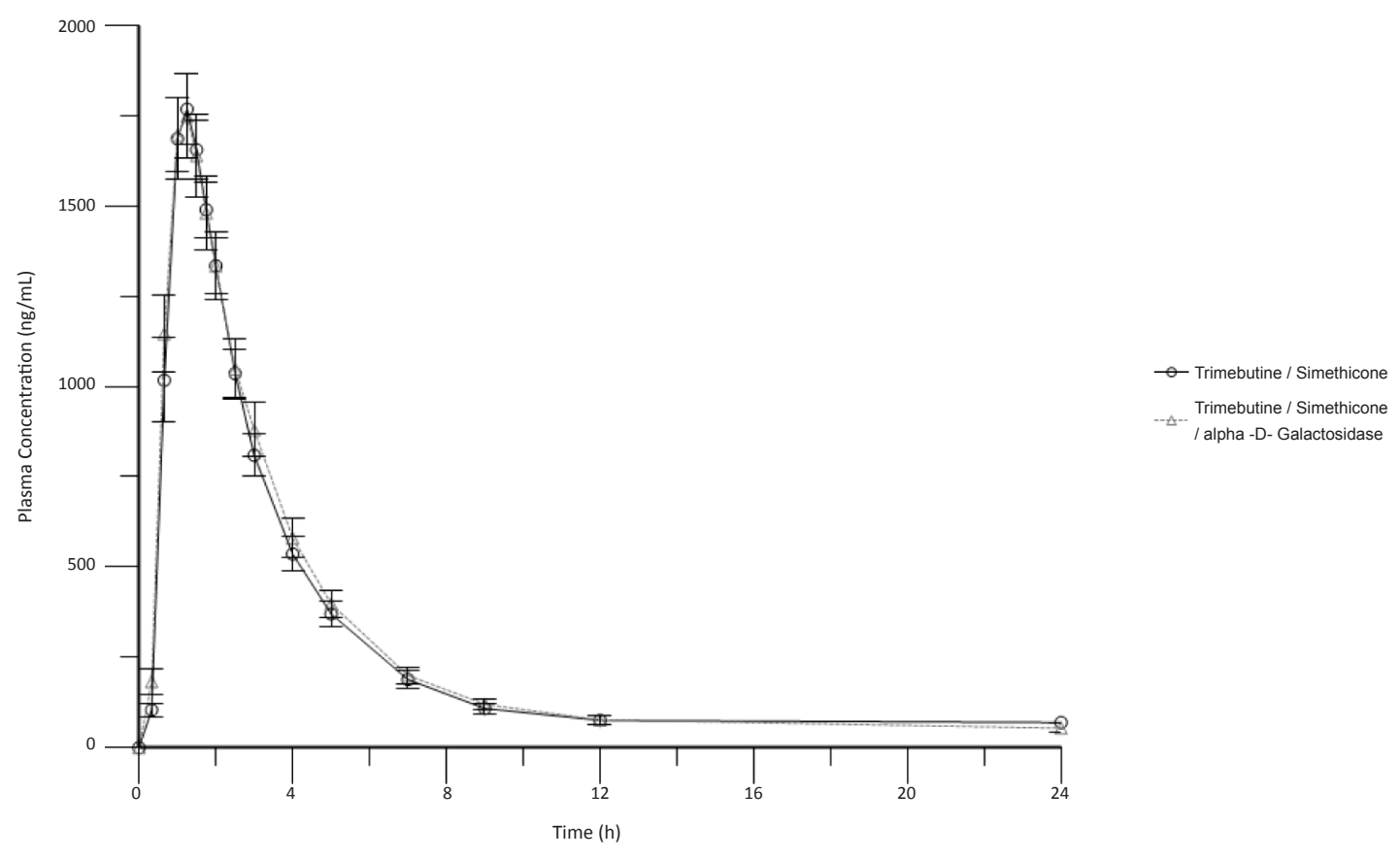

Figure 1: N-desmethyl-Trimebutine plasma concentration vs. time profile from 30 healthy volunteers after a single oral dose of $200 \mathrm{mg}$ Trimebutine maleate. Tablets containing 450 International Units (IU) of alpha-D-galactosidase (dashed line) were compared with tablets without the enzyme (continuous line). 
Citation: Peñaloza-Becerra CA, Ortega-Escamilla E, Vasquez-Vasquez JE, Marcelín-Jiménez G, P.Angeles-Moreno AC, et al. (2017) Alpha-DGalactosidase does not Interfere with Trimebutine Oral Pharmacokinetics in Mexican Healthy Volunteers. J Bioequiv Availab 9: $447-451$. doi: $10.4172 / j \mathrm{jbb} .1000342$

\begin{tabular}{|c|c|c|c|c|c|c|}
\hline \multirow{2}{*}{ Parameter } & \multicolumn{3}{|c|}{ Without alpha-GAL (Reference product) } & \multicolumn{3}{|c|}{ With alpha-GAL (Test product) } \\
\hline & Mean & SD & CV \% & Mean & SD & CV \% \\
\hline $\mathrm{T}_{\max }(\mathrm{h})$ & 1.283 & 0.327 & 25.442 & 1.32 & 0.538 & 40.743 \\
\hline$k_{e}\left(h^{-1}\right)$ & 0.311 & 0.097 & 31.071 & 0.298 & 0.096 & 32.103 \\
\hline$t_{1 / 2}(h)$ & 2.455 & 0.834 & 33.969 & 2.665 & 1.205 & 45.205 \\
\hline MRT (h) & 3.469 & 0.687 & 19.811 & 3.71 & 0.957 & 25.787 \\
\hline $\mathrm{C}_{\max }(\mathrm{ng} / \mathrm{mL})$ & $1,882.079$ & 548.692 & 29.154 & $1,926.538$ & 620.355 & 32.201 \\
\hline $\mathrm{AUC}_{0-\mathrm{t}}(\mathrm{ng} \mathrm{h} / \mathrm{mL})$ & $5,584.002$ & $2,394.599$ & 42.883 & $5,883.644$ & $2,615.918$ & 44.461 \\
\hline $\mathrm{AUC}_{0-\infty}(\mathrm{ng} \mathrm{h} / \mathrm{mL})$ & 5814.4 & $2,469.99$ & 42.481 & $6,124.035$ & $2,664.918$ & 43.516 \\
\hline $\mathrm{AUC}_{\text {ext }}(\%)$ & 4.09 & 1.308 & 31.977 & 4.177 & 2.413 & 57.767 \\
\hline
\end{tabular}

GAL: Alfa-Galactosidase; AUC $_{\text {ext }}$ : Area Under the Curve Extrapolated; MRT: Mean Residence Time; SD: Standard Deviation; CV: Coefficient of Variation Table 2: Pharmacokinetic parameters of nor-Trimebutine with and without Alpha-Galactosidase (Alpha-GAL).

\begin{tabular}{|c|c|c|c|c|c|c|}
\hline \multirow{2}{*}{ Parameter } & \multirow{2}{*}{ (\%) Intrasubject CV } & \multirow{2}{*}{ (\%) Ratio of geometric means } & \multicolumn{2}{|c|}{$90 \%$ Confidence Intervals } & \multicolumn{2}{|c|}{ Schuirmann test } \\
\hline & & & Lower & Upper & Prob 80 & Prob 125 \\
\hline $\mathrm{C}_{\max }(\mathrm{ng} / \mathrm{mL})$ & 13.781 & 101.953 & 95.992 & 108.284 & 0.00000 & 0.00000 \\
\hline $\mathrm{AUC}_{0-\mathrm{t}}\left(\mathrm{ng}{ }^{*} \mathrm{~h} / \mathrm{mL}\right)$ & 11.671 & 105.485 & 100.232 & 111.015 & 0.00000 & 0.00000 \\
\hline $\mathrm{AUC}_{0-\infty}\left(\mathrm{ng}^{*} \mathrm{~h} / \mathrm{mL}\right)$ & 11.272 & 105.606 & 100.520 & 110.948 & 0.00000 & 0.00000 \\
\hline
\end{tabular}

Table 3: Statistics bioequivalence of nor-Trimebutine with and without Alpha-Galactosidase (Alpha-GAL).

AG is an amylase-like enzyme possessing activity on alphagalactosidic bonds in non-digestible oligosaccharides, such as those found in the traditional Mexican diet, e.g., raffinose (from beans), stachyose (maize), and verbascose (wheat). These oligosaccharides are not completely digested in the human small bowel due to a natural absence of digestive enzymes capable of unfolding the bonds present in them. Resident flora fermentation, however, fully utilizes these compounds, producing hydrogen, methane, and carbon dioxide [6]. Thus, the rationale of including AG in formulations used in gas-prone diseases is directed toward attacking gas production, lowering the NDC reaching the terminal small bowel and large intestine, therefore diminishing gas production. This idea has been explored in other works, where AG from different origins were tested in animal models [6], children [14], and adults [3,15]; however, in those cases, AG was used alone and in different presentations. There is also evidence with respect to the development of possible probiotic products containing AG that are directed toward the relief of dietary restrictions or foodassociated gas production $[16,17]$.

The association of AG with Simethicone and Trimebutine has not, to our knowledge, been reported in the literature. Trimebutine is a widely used drug with prokinetic, peristalsis-modulating, and pain-relief properties that is useful in several conditions where gastrointestinal motility is disrupted. Its mechanism of action comprises a non-specific stimulation of opioid receptors, antimuscarinic actions, calcium channel regulation, and local anesthetic properties in gut. Trimebutine is absorbed in the stomach and the small bowel and suffers an extensive first-pass metabolism. Less than $4 \%$ remains unaltered, and the major metabolite $\mathrm{N}$-desmethyl-trimebutine is the responsible for its pharmacological activity. Metabolite exhibits a maximum plasma concentration around 1-2 $\mathrm{h}$, and meals does not modify its oral bioavailability. More than $70 \%$ of metabolite is excreted by urine during the first $24 \mathrm{~h}[18,19]$. It is frequently associated in commercial formulations with Simethicone. Simethicone is a silicon dioxide polymer (polydimethylsiloxane), which is deprived of systemic action. Its mechanism of action is that of inducing foam instability and lowering surface tension in gas bubbles within the intestine, thus favouring coalescence and relieving gas accumulation. It exerts no effect on nutrient absorption or gastrointestinal secretions [20]. The foam-dissolving properties of simethicone may improve the digestive activity of AG by facilitating its distribution along the intestine microenvironment.

In this work, we demonstrated that AG appears not to interfere with the pharmacokinetic features of the original formulation after the addition of AG. Trimebutine is the only absorbable drug in this presentation and the reference for comparing pharmacokinetic properties. This non-interfering behavior could be explained because trimebutine is absorbed in small bowel and rapidly reaches portal circulation. In the other hand, AG is an enzyme without systemic absorption and acts preferentially in large intestine, thus, lacking of pharmacokinetic parameters. As illustrated in Table 2, mean values of all of the parameters measured were practically not altered in both formulations, and these parameters are in agreement with those in the literature [18]. We found slight, but not statistically significant, increments in $\mathrm{CV} \%$ in the $\mathrm{C}_{\max }, \mathrm{t}_{1 / 2}$, and the MRT of test formulation. To rule out any interference and to detect any difference in time parameters, we performed a Wilcoxon test (there were no significant difference in any point). Thus, the slight increments could be explained by light changes in formulation due to manufacturing process. We also think that the intrinsic mechanism of absorption or delivery to metabolizing organs, as liver, was slightly modified, affording a broader range of time values, but with conservation of the mean values.

In conclusion, we demonstrated in this study that AG does not modify the pharmacokinetic parameters of Trimebutine in a formulation containing both the latter and Simethicone, providing support for releasing the commercial presentation and for testing in patients requiring this therapeutic alternative.

\section{References}

1. Bailey J, Carter NJ, Neher JO (2009) FPIN's clinical inquiries: effective management of flatulence. Am Fam Physician 79: 1098-1100.

2. National Center for Technological Excellence in Health (CENETEC) (2015) Irritable bowel diagnosis and treatment in adults: quick reference guide. In: IMSS (edn.) Catálaogo Master of Clinical Practice Guidelines-IMSS-042-08. General Health Council, Mexico, pp: 2-16.

3. Di Stefano M, Miceli E, Gotti S, Missanelli A, Mazzocchi S, et al. (2007) The effect of oral alpha-galactosidase on intestinal gas production and gas-related symptoms. Dig Dis Sci 52: 78-83.

4. Jhee OH, Lee YS, Shaw LM, Jeon YC, Lee MH, et al. (2007) Pharmacokinetic 
Citation: Peñaloza-Becerra CA, Ortega-Escamilla E, Vasquez-Vasquez JE, Marcelín-Jiménez G, P.Angeles-Moreno AC, et al. (2017) Alpha-DGalactosidase does not Interfere with Trimebutine Oral Pharmacokinetics in Mexican Healthy Volunteers. J Bioequiv Availab 9: $447-451$. doi: $10.4172 / j b b .1000342$

and bioequivalence evaluation of two formulations of $100 \mathrm{mg}$ trimebutine maleate (Recutin and Polybutin) in healthy male volunteers using the LC-MS/ MS method. Clin Chim Acta 375: 69-75.

5. Martínez-Vázquez MA, Vázquez-Elizondo G, González-González JA Gutiérrez-Udave R, Maldonado-Garza HJ, et al. (2012) Effect of antispasmodic agents, alone or in combination, in the treatment of Irritable Bowel Syndrome: systematic review and meta-analysis. Rev Gastroenterol Mex 77: 82-90

6. LeBlanc JG, Ledue-Clier F, Bensaada M, de Giori GS, Guerekobaya T, et al. (2008) Ability of Lactobacillus fermentum to overcome host alpha-galactosidase deficiency, as evidenced by reduction of hydrogen excretion in rats consuming soya alpha-galacto-oligosaccharides. BMC Microbiol 8: 22.

7. Cho KH, Choi YK, Kang JH, Choi HG, Yong CS, et al. (2010) Development of a novel combination tablet containing trimebutine maleate and mosapride citrate for the treatment of functional dyspepsia. Int J Pharm 400: 145-152.

8. World Medical Association (2002) World Medical Association Declaration of Helsinki: ethical principles for medical research involving human subjects. $J$ Postgrad Med 48: 206-208.

9. Shein-Chung C, Jen-Pei L (2009) Design and analysis of bioavailability and bioequivalence studies. Chapman and Hall/CRC, USA

10. Paquette JM, Rufiange $M$, lovu Niculita $M$, Massicotte $J$, Lefebvre $M$ et al. (2014) Safety, tolerability and pharmacokinetics of trimebutine 3-thiocarbamoylbenzenesulfonate (GIC-1001) in a randomized phase । integrated design study: single and multiple ascending doses and effect of food in healthy volunteers. Clin Ther 36:1650-1664

11. The Food and Drug Administration (FDA) (2013) Guidance for Industry: Bioanalytical Method Validation. FDA, USA p: 34

12. Medication, PPQU, Mexican official NOM-177-SSA1 (2013) Which establishes the tests and procedures to demonstrate that a drug is interchangeable.
Requirements to be met by the Authorized Third Parties performing the interchangeability tests. Requirements for carrying out the biocomparability studies. Requirements to be met by Authorized Third Parties, Research Centers or Hospital Institutions that perform the biocomparability tests. Ministry of Health, Mexico DF, pp: 17-128.

13. Hiyama T, Yoshihara M, Matsuo K, Kusunoki H, Kamada T, et al. (2007) Metaanalysis of the effects of prokinetic agents in patients with functional dyspepsia. J Gastroenterol Hepatol 22: 304-310.

14. Di Nardo G, Oliva S, Ferrari F, Mallardo S, Barbara G, et al. (2013) Efficacy and tolerability of $\alpha$-galactosidase in treating gas-related symptoms in children: a randomized, double-blind, placebo controlled trial. BMC Gastroenterol 13: 142

15. Di Pierro F, Bertuccioli A, Marini E, Ivaldi L (2015) A pilot trial on subjects with lactose and/or oligosaccharides intolerance treated with a fixed mixture of pure and enteric-coated $\alpha$ - and $\beta$-galactosidase. Clin Exp Gastroenterol 8: 95-100.

16. Liu X, Champagne CP, Lee BH, Boye JI, Casgrain M (2014) Thermostability of Probiotics and Their $\alpha-$ Galactosidases and the Potential for Bean Products. Biotechnol Res Int 2014: 472-723.

17. Patil AG, K PK, Mulimani VH, Veeranagouda Y, Lee K (2010) alpha-Galactosidase from Bacillus megaterium VHM1 and its application in removal of flatulencecausing factors from soymilk. J Microbiol Biotechnol 20: 1546-1554.

18. Khan I (2011) Determination of Active Metabolite Desmethyl Trimebutine Levels after a Single Dose of Trimebutine Tablets in Healthy Human Male Volunteers. Int J PharmTech Res 3: 1219-1226.

19. Lee HT, Kim BJ (2011) Trimebutine as a modulator of gastrointestinal motility. Arch Pharm Res 34: 861-864.

20. Brecević L, Bosan-Kilibarda I, Strajnar F (1994) Mechanism of antifoaming action of simethicone. J Appl Toxicol 14: 207-211. 